\title{
Percutaneous Screw Fixation of Adolescent Skier's Thumb. A Case Report and Review of the Literature
}

\section{Chatziravdeli Vasiliki*, Tsatlidou Maria, Vazakidis Polychronis, Abouhamdan Mahdi and Mpeletsiotis Anastasios}

$2^{\text {nd }}$ Orthopaedic Department, General Hospital of Thessaloniki "Papageorgiou”, Thessaloniki, Greece

*Corresponding Author: Chatziravdeli Vasiliki, $2^{\text {nd }}$ Orthopaedic Department, General Hospital of Thessaloniki "Papageorgiou”, Thessaloniki, Greece.
Received: March 29, 2021

Published: April 09, 2021

(C) All rights are reserved by Chatziravdeli

Vasiliki., et al.

\begin{abstract}
Adolescent skier's thumb is a common injury in this high activity level age group, that involves a Salter Harris (SH) III avulsion fracture with the ulnar collateral ligament (UCL) attached to it. Optimal fracture stabilisation therefore affects joint stability. Operative treatment for large, displaced fragments involves open reduction with the use of Kirchner wires, screws, bone anchors or pull out sutures in order to achieve anatomic restoration of the epiphyseal fracture with respect to the physis. Our case report involves percutaneous reduction of an SH III fracture of the proximal phalanx of the thumb in an adolescent with the joystick technique and cannulated compression screw fixation without crossing the physis. This method provided stable fixation and allowed for early commencement of range of motion exercises and early return to previous activities. The radiographic evidence after 18 months demonstrated fracture consolidation with no malalignment and no residual thumb instability. The best treatment strategy should be based on fracture characteristics, joint stability and patient's activity level and preferences. It is our suggestion that percutaneous screw fixation by closed means should be attempted for reducible fragments prior to open reduction techniques, to avoid disturbing local anatomy and prevent scarring.
\end{abstract}

Keywords: Adolescent; Skier's Thumb; Percutaneous; SH III Avulsion; UCL; Proximal Phalanx

\section{Introduction}

Traumatic rupture of the ulnar collateral ligament (UCL) of the thumb, also known as skier's or Gamekeeper's thumb is an injury that commonly affects the adult population. In adolescents with open physis, the same mechanism of injury, which is forced abduction with or without hyperextension of the proximal phalanx of the thumb, rarely leads to UCL tear. Instead, the energy dissipates through the growth plate leading to a Salter Harris III epiphyseal fracture with the UCL attached to it [1]. In a prospective study of 360 children by Vadivelu., et al. 2006, it was demonstrated that the most commonly injured site in older children, was the proximal phalanx, with $23 \%$ involving the thumb [2]. In the presence of SH III fractures, treatment should follow the principles of intraarticular fractures in the growing population and require special consideration as they are more frequently associated with growth disturbance and post-traumatic arthritis latter in life. For proximal phalanx epiphyseal fractures more than $2 \mathrm{~mm}$ of displacement, $>1$ $\mathrm{mm}$ articular step-off and $>25 \%$ of epiphyseal involvement operative treatment is indicated $[3,4]$. 


\section{Case Report}

A 14 year-old male adolescent was referred from a District Hospital to our Unit after suffering a traumatic Salter Harris III (SH) fracture of the base of the proximal phalanx of the right thumb. The patient was right hand dominant and had a fall on his outstretched hand with forceful thumb abduction, which resulted in the above injury. Using the Xero Viewer computer system (AgfaHealthCare) which can accurately calculate distance between to points and perform angle measurements, more than $>2 \mathrm{~mm}$ of displacement, 30 degrees of rotation and $40 \%$ of articular involvement of the fragment was calculated. After a failed attempt of closed reduction at the District Hospital he was referred to our Hospital for surgical treatment. The patient was neurovascularly intact with pain over the fracture site but abduction of the right thumb could not be evaluated due to discomfort (Image 1).

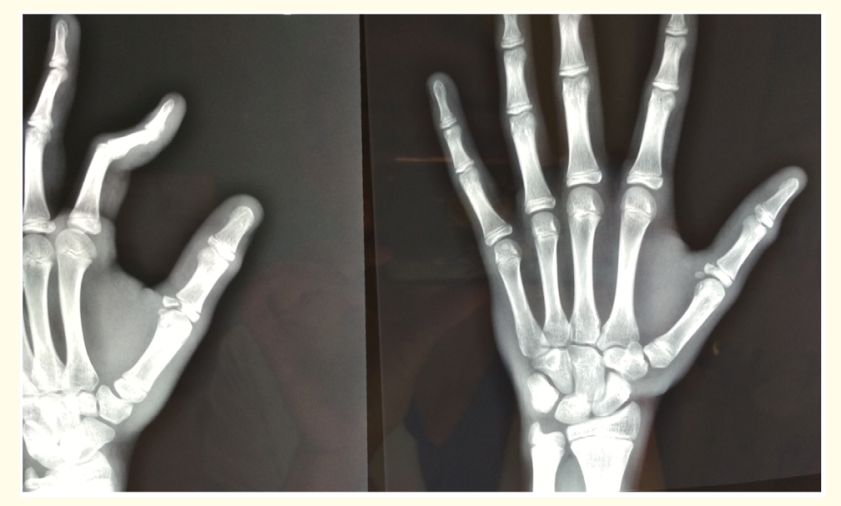

Image 1: Right hand X-ray demonstrating Salter Harris III avulsion fracture of the proximal phalanx of the thumb.

The width of the proximal phalanx epiphysis was measured 4 $\mathrm{mm}$ and therefore the use of a single cannulated screw was the fixation method chosen. Under fluoroscopy with the hand on an arm table, a guide wire was used to percutaneously engage the fracture fragment and reduce it with the joystick technique. When targeting the fragment with the guide wire, effort was made to engage it in the middle of its width and during the reduction the trajectory of the wire was not horizontal but slightly angulated from proximal to distal in order to avoid penetrating into the metacarpophalangeal (MCP) joint and the physis (Image 2). A single titanium cannulated headless compression screw $1.5 \mathrm{~mm}$ was passed through the guide wire for fracture fixation with a stab incision.

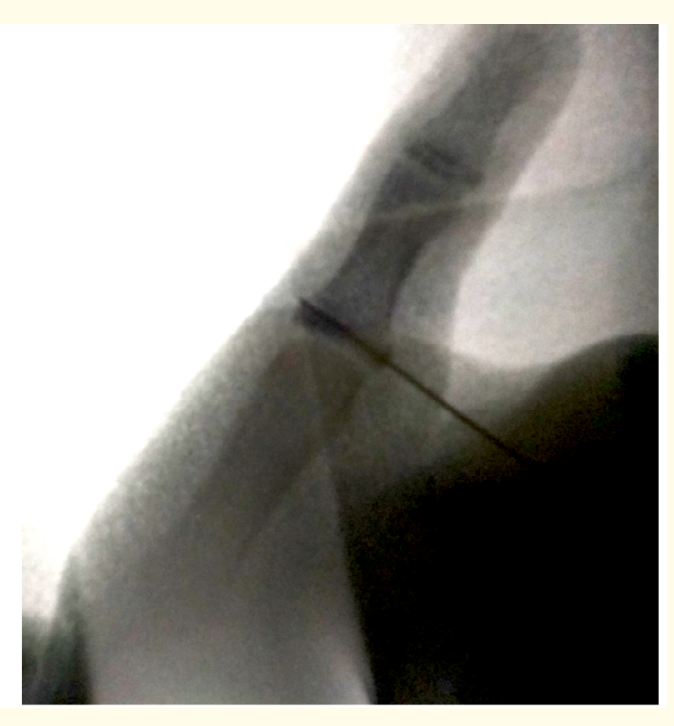

Image 2: Intraoperative X-ray with fracture reduction through the guidewire.

The patient was fitted with a removable thumb spica splint for 3 weeks with progressive range of motion exercises commenced at 10 days post-operatively. He was able to fully resume his previous activities without restrictions at 6 weeks. At 18 months follow up, his X-ray revealed physeal closure of the hand phalanx and metacarpal bones and complete fracture healing with no malalignment. Pintch strength, grip and range of movement was equivalent to the contralateral side. No stiffness or pain and no instability of the UCL compared to the uninjured side was noted (Image 3 and 4).

\section{Discussion}

Adolescent avulsion fracture of UCL is not an uncommon injury but there are no clear recommendations to guide clinical decision regarding conservative versus operative treatment. Conservative treatment yields good, predictable results in cases with minimal fragment displacement and if the MCP joint is stable [5,6]. Studies that involve only participants with open physis are sparse in the literature and most of the information come from studies on adults [7-9]. In a recent study by Minh N Q Huynh., et al. 2020, surgical treatment demonstrated quicker healing rates but overall conservative methods even for fractures that fitted the criteria for surgery had good healing and functional results but more time to union 


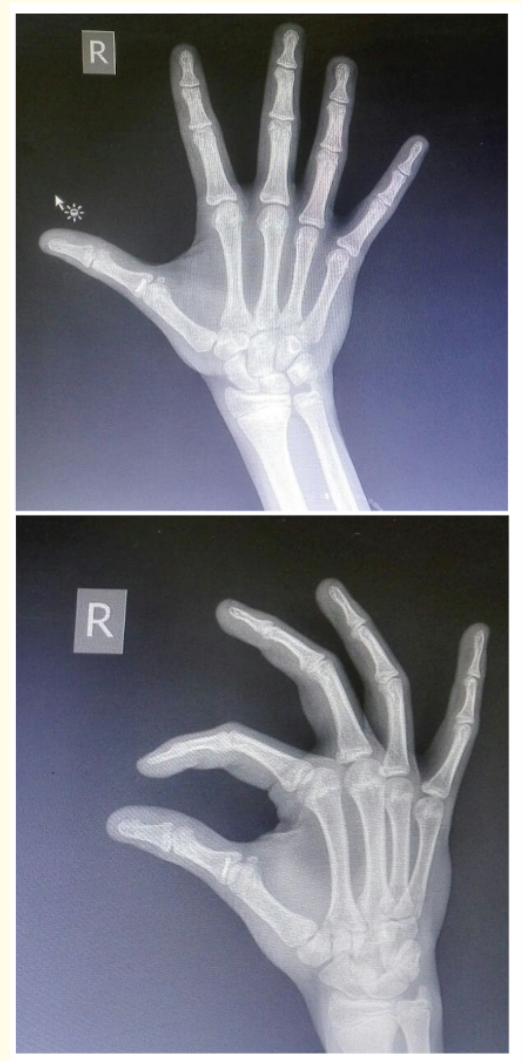

Image 3: Fracture consolidation at 18 months follow up.
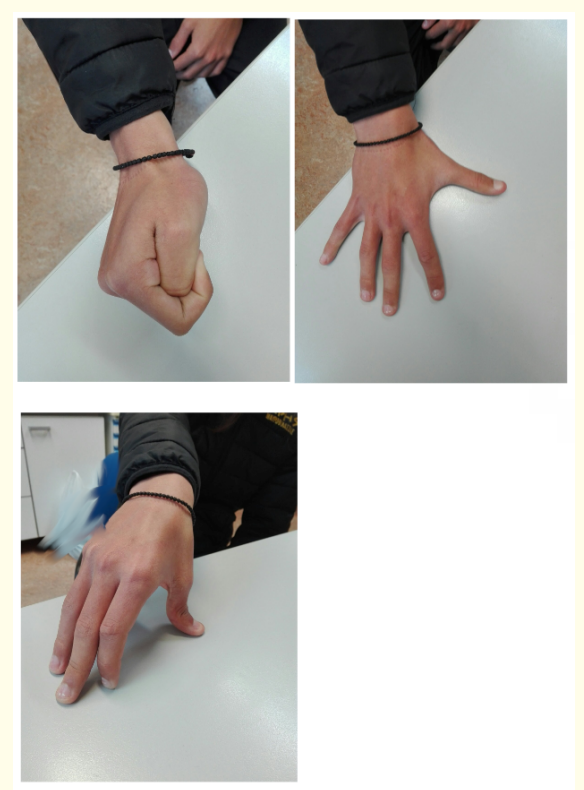

Image 4: Clinical evaluation at 18 months follow up.
[10]. The same study also demonstrated good results for patients with delayed surgery due to failed conservative methods.

There are several methods of surgical treatment described in the literature like open reduction and Kirchner wire (KW) stabilisation, screw fixation, bone anchor, pull out suture [11]. Fractures that are displaced and rotated usually require open reduction in order to restore stability and joint congruency $[3,12]$. The epiphyseal fracture may get displaced outside the adductor aponeurosis (Stener lesion) preventing healing or leading to malunion $[13,14]$.

Our method of choice was to use percutaneous screw fixation in order to avoid an open approach, which could lead to scarring or wound related complications. KW fixation was rejected because they cannot achieve compressive fixation with the risk of pin track infection or malunion [15]. Due to the fact that our patient was an adolescent with open phalangeal physis, we aimed to avoid penetrating the physis and the MCP joint with our screw. In order to avoid damaging neurovascular structures of the thumb, we used the "safe corridors" as described by C Rex., et al. 2015 [16] and in case that would not be applicable a "nick and spread" technique would be utilised, which was not deemed necessary in our case. By using compression screw combined with percutaneous reduction, we achieved good fracture fixation with minimal soft tissue injury and a quick patient recovery.

\section{Conclusion}

In general, there are no large scale studies in the skeletally immature population, with clear-cut criteria that can guide a clinician's decision. The best treatment strategy should be based on fracture characteristics, joint stability and patient's activity level and preferences. It is our suggestion that percutaneous screw fixation by closed means is a viable solution for adolescent patients with skier's thumb lesions and leads to early mobilisation and excellent results. It should be attempted for reducible fragments prior to open reduction techniques, to avoid disturbing local anatomy and prevent scarring.

\section{Informed Consent}

Informed patient's consent was obtained for the purpose of the current study.

\section{Conflict of Interest}

The authors declare that they have no conflicts of interest.

\section{Financial Support}

This work received no financial support. 


\section{Bibliography}

1. J Graham and ET O’Brien. "Fractures and Dislocations of the Hand and Carpus in Children". Fractures in Children ( $4^{\text {th }}$ Edition), edited by J.H. Beaty C.A. Rockwood Jr, K.E. Wilkins, $4^{\text {th }}$ edition. Lippincott-Raven (1996): 432-434.

2. Vadivelu Ramanan., et al. "Hand Injuries in Children: A Prospective Study". Journal of Pediatric Orthopedics 26.1 (2006): 29-35.

3. Kozin Scott H. "Fractures and Dislocations along the Pediatric Thumb Ray”. Hand Clinics 22.1 (2006): 19-29.

4. Torre BA. "Epiphyseal Injuries in the Small Joints of the Hand". Hand Clinics 4.1 (1988): 113-121.

5. Landsman JC., et al. "Splint Immobilization of Gamekeeper's Thumb". Orthopedics 18.12 (1995): 1161-1165.

6. Ritting Andrew W., et al. "Ulnar Collateral Ligament Injury of the Thumb Metacarpophalangeal Joint". Clinical Journal of Sport Medicine: Official Journal of the Canadian Academy of Sport Medicine 20.2 (2010): 106-112.

7. Madan Simerjit Singh., et al. "Injury to Ulnar Collateral Ligament of Thumb". Orthopaedic Surgery 6.1 (2014): 1-7.

8. Rhee Peter C., et al. "Management of Thumb Metacarpophalangeal Ulnar Collateral Ligament Injuries". The Journal of Bone and Joint Surgery American 94.21 (2012): 2005-2012.

9. Goth D and P Haussmann. "[Surgical treatment of the ruptured ulnar collateral ligament of the metacarpophlangeal joint of the thumb]". Handchirurgie 11.1 (1979): 61-64.

10. Huynh Minh NQ., et al. "A Comparison of Conservative and Operative Management of Thumb Ulnar Collateral Ligament Avulsion Fractures in Children". Hand 15.6 (2020): 812-817.

11. Wolfe S PW., et al. "Green's Operative Hand Surgery". Elsevier (2010).

12. Zlotolow Dan A and Scott H Kozin. "Hand and Wrist Injuries in the Pediatric Athlete". Clinics in Sports Medicine 39.2 (2020): 457-479.

13. White GM. "Ligamentous Avulsion of the Ulnar Collateral Ligament of the Thumb of a Child". The Journal of Hand Surgery 11.5 (1986): 669-672.
14. Beutel Bryan G., et al. "The Stener Lesion and Complete Ulnar Collateral Ligament Injuries of the Thumb A Review". Bulletin of the Hospital for Joint Disease 77.1 (2019): 11-20.

15. AO Foundation. “General K Wire Principles” (2021).

16. Rex C., et al. "Safe Corridors for K-Wiring in Phalangeal Fractures". Indian Journal of Orthopaedics 49.4 (2015): 388-392.

\section{Assets from publication with us}

- Prompt Acknowledgement after receiving the article

- Thorough Double blinded peer review

- Rapid Publication

- Issue of Publication Certificate

- High visibility of your Published work

Website: www.actascientific.com/

Submit Article: www.actascientific.com/submission.php

Email us: editor@actascientific.com

Contact us: +919182824667 